\title{
EDITORIAL
}

\section{Cervical cancer prevention and detection in focus}

Luiz Martins Collaço

The fact that approximately 500 thousand women develop new cases of cervical cancer ${ }^{(1)}$ per year worldwide is alarming and gives rise to great concern to the scientific community about a preventable disease.

The discovery that human papillomavirus (HPV) is associated with genesis of precursory lesions and the invasive cervical carcinoma, promoted several changes on early prevention and detection approach of this lesion. Despite the cytopathological exam (Pap smear) be a recommended technique for mass prevention due to its historical and cost-benefit aspects, it has been extensively debated the exam limitations on respect of sensitivity, specificity, and reproducibility ${ }^{(1)}$. As a result of progress in the study of lesions genesis and behavior, new technologies are partnering in an attempt to contain the spread of the disease. Among them are the molecular tests, especially the hybrid capture for HPV, and the prophylactic vaccines against the virus.

The molecular test for HPV by hybrid capture, used as primary or complementary test for the investigation of changes found in cytology, has been used as a strategy in screening programs since the Food and Drug Administration (FDA) approved it as a primary or complementary test to altered cytology ${ }^{(1)}$. This strategy, associated with Pap smear test provides a better selection of cases to be followed with morphological study (cytology), and extends the screening range reducing costs in medium and long term ${ }^{(4)}$.

Prophylactic vaccination against HPV, now established in Brazil as a method of combating the disease, is an alternative with great promise for promoting a drastic reduction in the number of cervical cancer, and try to control the spread of the diseases, which is already happening in other countries, where has been used for a longer time. The future prospects is that the association of the vaccine with the triennial cytology may reduce in $94 \%$ the risk of cervical cancer ${ }^{(1)}$.

On this line of discussion of methods and their applicability in relation to HPV-induced lesions, the present issue of the Brazilin Journal of Pathology and Laboratory Medicine (Jornal Brasileiro de Patologia e Medicina Laboratorial [JBPML]) brings the article "HPV detection using primers MY09/MY11 and GP5+/GP6+ in patients with cytological and/or colposcopic changes"(3), which discuss the applicability of polymerase chain reaction (PCR) as another element to be analyzed, since it has been the most performed technique by several areas of molecular diagnosis ${ }^{(2)}$. We are certain that the discussion of this topic will provide the reader a further element for consideration on the possibilities of enhancement of the methods to help the prevention and detection of cervical cancer.

\section{REFERENCES}

1. COLLACO, L. M.; ZARDO, L. Cytologic screening programs. In: BIBBO, M.; WILBUR, D. Comprehensive cytopathology. 3. ed. Philadelphia, 2008. Cap 3; p. 47-57.

2. GRAVITT, P. E. et al. Improved amplification of genital human papillomaviruses.J Clin Microbiol, v. 38, n. 1, p. 357-61, 2000.

3. VENCESLAU, E. M. et al. HPV detection using primers MY09/MY11 and GP5+/GP6+ in patients with cytologic and/or colposcopic changes. JBPML, v. 50, n. 4, p. 280-85, 2014.

4. YOSHIDA, T. et al. Comparison of self-collected and clinician-collected materials of cervical cytology and human papilomavirus genotyping: analysis by linear array assay. Acta Cytol, v. 50, p. 106-12, 2011.

Pathologist; MsC-PhD in Medicine; professor of Anatomic Pathology at Universidade Federal do Paraná (UFPR) and the Faculdade Evangélica do Paraná; member of International Academy of Cytology; past president of the Sociedade Brasileira de Citopatologia. 